\title{
The role of CD44 in the acute and resolution phase of the host response during pneumococcal pneumonia
}

\author{
Gerritje JW van der Windt ${ }^{1,2}$, Arie J Hoogendijk ${ }^{1,2}$, Alex F de $\operatorname{Vos}^{1,2}$, Marjolein E Kerver ${ }^{1,2}$, Sandrine Florquin ${ }^{3}$ \\ and Tom van der Poll,
}

Streptococcus pneumoniae is the most prevalent pathogen causing community-acquired pneumonia. CD44 is a transmembrane adhesion molecule, expressed by a wide variety of cell types, that has several functions in innate and adaptive immune responses. In this study, we tested the hypothesis that CD44 is involved in the host response during pneumococcal pneumonia. On intranasal infection with a lethal dose of S. pneumoniae CD44-knockout (KO) mice showed a prolonged survival when compared with wild-type mice, which was accompanied by a diminished pulmonary bacterial growth and reduced dissemination to distant body sites. Whereas, proinflammatory cytokine responses and lung pathology were not affected, CD44 deficiency resulted in increased early neutrophil influx into the lung. In separate experiments, we confirmed a detrimental role of CD44 in host defense against pneumococci during sublethal pneumonia, as demonstrated by an improved capacity of CD44 KO mice to clear a low infectious dose. In addition, CD44 appeared important for the resolution of lung inflammation during sublethal pneumonia, as shown by histopathology of lung tissue slides. In conclusion, we show here that CD44 facilitates bacterial outgrowth and dissemination during pneumococcal pneumonia, which in lethal infection results in a prolonged survival of CD44 KO mice. Moreover, during sublethal pneumonia CD44 contributes to the resolution of the inflammatory response.

Laboratory Investigation (2011) 91, 588-597; doi:10.1038/labinvest.2010.206; published online 17 January 2011

KEYWORDS: bacteria; infection; inflammation; lung; Streptococcus pneumoniae

Bacterial pneumonia is a common and serious illness that is a leading cause of morbidity and mortality. ${ }^{1}$ Streptococcus pneumoniae (the pneumococcus) is the most prevalent microorganism in community-acquired pneumonia, responsible for an estimated 10 million deaths annually worldwide. $^{2,3}$ The increasing incidence of antibiotic resistance in this pathogen stresses the importance to expand our knowledge of the host defense mechanisms that influence the outcome of pneumococcal pneumonia. ${ }^{4}$

CD44 is a transmembrane adhesion molecule that is expressed by a wide variety of cell types, including leukocytes and parenchymal cells. ${ }^{5,6}$ It has several functions in innate and adaptive immune responses, such as involvement in cellular adhesion and migration, lymphocyte/monocyte activation and proliferation, and cell-mediated cytotoxicity in natural killer cells. ${ }^{5-7}$ In accordance, CD44 has been shown to have an eminent role in the (sub)acute inflammatory response to both infectious and sterile stimuli. ${ }^{8-11} \mathrm{CD} 44$ is also involved in the resolution of inflammation, as demonstrated by in vivo models of non-infectious lung injury induced by bleomycin or lipopolysaccharide (LPS); in these studies CD44 deficiency resulted in prolonged accumulation of inflammatory cells and a persistent rise in the levels of the main CD44 ligand hyaluronic acid (HA). ${ }^{8,12}$ Furthermore, during infection, CD44 may influence host defense by affecting phagocytosis. For instance, our laboratory showed that CD44 on murine macrophages mediates phagocytosis of Mycobacterium tuberculosis, ${ }^{13}$ whereas phagocytosis of Staphylococcus aureus is induced on the activation of CD44 on human neutrophils by HA or monoclonal antibodies. ${ }^{14}$

To the best of our knowledge, only one study investigated the role of CD44 during bacterial pneumonia, focusing on the acute response during respiratory tract infection caused by Escherichia coli or S. pneumoniae. ${ }^{9}$ In that previous investigation,

\footnotetext{
${ }^{1}$ Center for Infection and Immunity Amsterdam, Academic Medical Center, University of Amsterdam, Amsterdam, The Netherlands; ${ }^{2}$ Center for Experimental and Molecular Medicine, Academic Medical Center, University of Amsterdam, Amsterdam, The Netherlands and ${ }^{3}$ Department for Pathology, Academic Medical Center, University of Amsterdam, Amsterdam, The Netherlands

Correspondence: Professor T van der Poll, MD, PhD, Center for Infection and Immunity Amsterdam, Academic Medical Center, University of Amsterdam, Meibergdreef 9, Room G2-130, Amsterdam 1105 AZ, The Netherlands.

E-mail: t.vanderpoll@amc.uva.nl

Received 10 May 2010; revised 12 September 2010; accepted 22 September 2010
} 
CD44-knockout $(\mathrm{KO})$ mice were reported to demonstrate an enhanced inflammatory response in their lungs at $6 \mathrm{~h}$ after infection with E. coli, as reflected by an increased influx of neutrophils and more edema; the pulmonary response to S. pneumoniae was not influenced by $\mathrm{CD} 44$ deficiency during the first $6 \mathrm{~h}^{9}{ }^{9}$ The role of CD44 during more prolonged lung infection (ie, beyond $6 \mathrm{~h}$ ) was not studied in either model. ${ }^{9}$ The host response to bacterial pneumonia can be roughly divided in an acute response, seeking to kill the pathogens that try to invade the lower airways, and a late response, with the primary aim to clear the inflammatory reaction and restore tissue homeostasis. ${ }^{15,16}$ In this study, we sought to investigate the function of CD44 during S. pneumoniae-induced pneumonia, comparing both the induction of the acute innate immune response after lethal infection (during the first $48 \mathrm{~h}$ after infection) and the resolution of inflammation after sublethal infection in CD44 KO and wild-type (WT) mice (4-10 days after infection).

\section{MATERIALS AND METHODS \\ Mice}

C57BL/6 WT mice (9 to 12 week old) were purchased from Harlan Sprague Dawley (Horst, The Netherlands). CD44 $\mathrm{KO}$ mice on a C57BL/6 background (kindly provided by Dr A Berns, Netherlands Cancer Institute, Amsterdam, The Netherlands ${ }^{17}$ ) were bred in the animal facility of the Academic Medical Center (Amsterdam, The Netherlands). For all experiments age and sex matched animals were used.

\section{Study Design}

The Animal Care and Use Committee of the University of Amsterdam approved all experiments. Pneumonia was induced by intranasal inoculation of $10^{4}$ or $10^{2}$ colony-forming units (CFUs) of S. pneumoniae serotype 3 (ATCC 6303; American Type Culture Collection, Rockville, MD, USA), as described previously. ${ }^{18-20}$ All procedures regarding collection and processing of samples were carried out as described. ${ }^{18-20}$ Briefly, after 6,24 or $48 \mathrm{~h}$, and 4 or 10 days mice ( $n=7$ to 12 per strain at each time point) were killed, blood was drawn in heparin containing tubes, lungs were lavaged and organs were removed aseptically and homogenized in five volumes of sterile $0.9 \% \mathrm{NaCl}$ using a tissue homogenizer (Biospec Products, Bartlesville, OK, USA). To determine bacterial loads, 10 -fold dilutions of blood, lung and spleen homogenates were plated on blood agar plates and incubated at $37^{\circ} \mathrm{C}$ for $16 \mathrm{~h}$. For bronchoalveolar lavage (BAL), the trachea was exposed through a midline incision and cannulated with a sterile 22-gauge Abbocath-T catheter (Abott, Sligo, Ireland). BAL was performed by instilling three $0.3 \mathrm{ml}$ aliquots of sterile phosphate buffered saline. Cell counts were determined for each BAL fluid (BALF) sample in a hemocytometer (Beckman Coulter, Fullerton, CA, USA) and differential cell counts were performed on cytospin preparations stained with Giemsa stain (Diff-Quick; Dade Behring AG,
Düdingen, Switzerland). For survival studies, mice ( $n=11$ to 12 per strain) were monitored for 10 days after infection.

\section{Assays}

Myeloperoxidase (MPO), tumor necrosis factor (TNF)- $\alpha$, interleukin (IL)-6, IL-1 $\beta$, keratinocyte-derived cytokine (KC), macrophage inflammatory protein (MIP)-2, LPSinduced CXC chemokine (LIX) and HA were measured by ELISA using matched antibody pairs according to the manufacturer's instructions (MPO; Hycult, Uden, The Netherlands, others; R\&D Systems, Abingdon, UK). TNF- $\alpha$ and IL- 6 in plasma were measured by cytometric bead array multiplex assay in accordance with the manufacturer's recommendations (BD Biosciences, San Jose, CA, USA; detection limits: $2.5 \mathrm{pg} / \mathrm{ml}$ ).

\section{Lung Histology}

Lungs were harvested, fixed in $10 \%$ buffered formalin for $24 \mathrm{~h}$, and embedded in paraffin. Hematoxilin- and eosinstained slides were coded and scored from 0 (absent) to 4 (severe) for the following parameters: interstitial inflammation, endothelialitis, bronchitis, edema, thrombi, pleuritis and percentage of the lung surface demonstrating confluent (diffuse) inflammatory infiltrate by a pathologist blinded for groups. The total 'lung inflammation score' was expressed as the sum of the scores for each parameter, the maximum being 28 .

\section{Statistical Analysis}

All data are expressed as mean \pm s.e.m. Comparisons between groups were analyzed by Mann-Whitney $U$-test using GraphPad Prism version 4.0, GraphPad Software (San Diego, CA, USA). Survival was compared by log-rank test. Comparisons of the number of positive and negative cultures were done using $\chi^{2}$-tests. A value of $P<0.05$ was considered statistically significant.

\section{RESULTS CD44 Accelerates Mortality During Lethal Pneumonia} As a first attempt to determine the role of CD44 in host defense against severe pneumococcal pneumonia, we infected WT and CD44 KO mice with $10^{4} \mathrm{CFU}$ of $S$. pneumoniae, a bacterial dose that causes pulmonary sepsis and lethality in normal immunocompetent mice, ${ }^{19,21}$ and followed them for 10 days. In both groups, the first deaths occurred after about 2.5 days; however, before the end of the fifth day after infection, all WT mice had succumbed, whereas at that time point approximately half of the $\mathrm{CD} 44 \mathrm{KO}$ mice were still alive. Hence, CD44 KO mice had a clear survival benefit when compared with WT mice $(P=0.001$; Figure 1a).

\section{CD44 Facilitates Bacterial Outgrowth and Dissemination During Lethal Pneumonia}

Next we questioned whether CD44 affects the antibacterial response against this Gram-positive pathogen. Therefore, we 

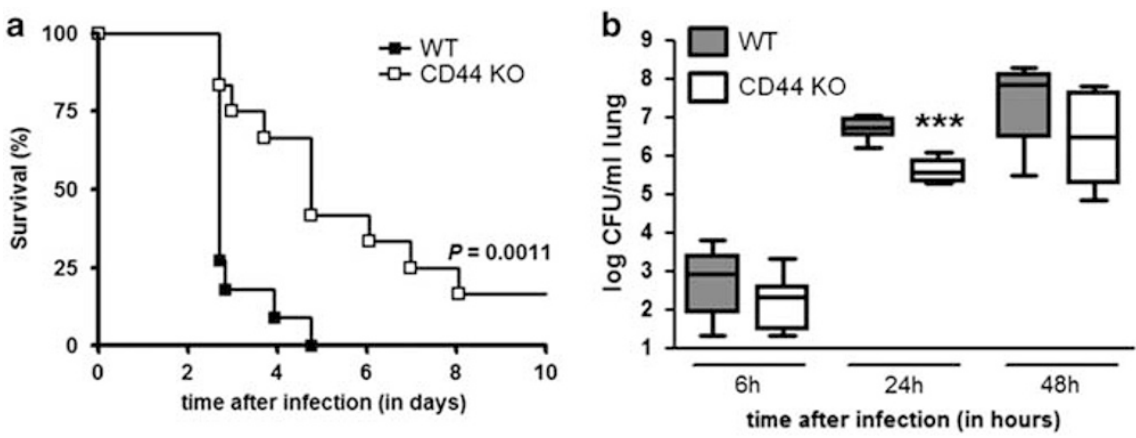

Figure 1 CD44 KO mice show delayed mortality and reduced bacterial outgrowth after infection with a lethal dose of S. pneumoniae. (a) Percentage survival of WT (closed symbols) and CD44 KO (open symbols) mice after intranasal infection with $10^{4}$ CFU of S. pneumoniae, followed for 10 days. $n=11$ to 12 mice per group. P-value indicates the difference between groups. (b) Bacterial loads determined in lung homogenates from WT (closed symbols) and CD44 KO (open symbols) mice at 6, 24 and $48 \mathrm{~h}$ of infection with $10^{4} \mathrm{CFU}$ of S. pneumoniae. Data are expressed as box-and-whisker diagrams depicting the smallest observation, lower quartile, median, upper quartile and largest observation; $n=8$ mice/group. ${ }^{* *} P<0.001$ versus WT at the same time point.

determined bacterial outgrowth in whole-lung homogenates, blood and spleen homogenates of CD44 KO and WT mice at 6,24 and $48 \mathrm{~h}$ after infection, time points representative of both the early and late host response (ie, just before the first deaths occurred). At $6 \mathrm{~h}$ post infection, bacterial numbers were similar in lung homogenates prepared from CD44 KO and WT mice (Figure 1b). At $24 \mathrm{~h}$, however, pulmonary bacterial loads were significantly reduced in $\mathrm{CD} 44 \mathrm{KO}$ mice $(P<0.001$ versus WT mice); this difference was not detectable anymore $48 \mathrm{~h}$ after infection (Figure 1b). Remarkably, whereas none of the mice in either group showed positive blood or spleen cultures at $6 \mathrm{~h}$ post infection, CD44 KO mice showed a significantly diminished dissemination of the infection at $24 \mathrm{~h}$, as reflected by the fact that $S$. pneumoniae could be cultured from blood of only 1/8 CD44 KO mice versus $8 / 8$ WT mice and from spleen homogenates of $3 / 8$ CD44 KO versus $8 / 8$ WT mice $(P<0.001$ and $P<0.01$, respectively). At $48 \mathrm{~h}$ after infection, blood and spleen cultures were positive in all $\mathrm{CD} 44 \mathrm{KO}$ and WT mice, and quantitatively not different between the two mouse strains (data not shown). Together these results suggest that CD44 facilitates the early bacterial outgrowth and dissemination during severe pneumococcal pneumonia, which might at least partially explain the delayed lethality of CD44 $\mathrm{KO}$ mice.

\section{CD44 does not Impact on Lung Pathology During Lethal Pneumonia}

This model of lethal pneumococcal pneumonia is associated with considerable lung pathology, characterized by interstitial inflammation together with endothelialitis, bronchitis, edema and pleuritis. ${ }^{21,22}$ To analyze the role of CD44 herein, we semiquantitatively scored histopathology slides prepared from lungs harvested at 24 or $48 \mathrm{~h}$ after infection using the semiquantitative scoring system described in the Materials and Methods section. The extent of lung inflammation did not differ between CD44 KO and WT mice at either time point (Figure 2a-f).

\section{CD44 does not Affect Proinflammatory Cytokine Levels During Lethal Pneumonia}

Cytokines have a pivotal role in host defense against pneumococcal pneumonia. ${ }^{3,23}$ Therefore, we measured the levels of proinflammatory cytokines (TNF- $\alpha$, IL- 6 and IL- $1 \beta$ ) in lungs obtained from CD44 KO and WT mice at 6,24 or $48 \mathrm{~h}$ after infection. The concentrations of all mediators were similar in CD44 KO and WT mice at all time points (Table 1). In addition, we measured TNF- $\alpha$ and IL-6 levels in plasma. At $6 \mathrm{~h}$ after infection, these parameters were not detectable in plasma yet. At $24 \mathrm{~h}$ after infection, TNF- $\alpha$ and IL-6 levels were significantly decreased in plasma from CD44 KO mice as compared with the concentrations measured in WT mice. At $48 \mathrm{~h}$, plasma TNF- $\alpha$ and IL-6 levels were still lower in CD44 KO mice, although not significantly anymore (Table 1 ).

\section{CD44 Deficiency Results in Enhanced Early Neutrophil Recruitment into the Lung on S. Pneumoniae Infection} CD44 has been implicated as an important mediator of leukocyte trafficking during infection and inflammation..$^{8-10,24,25}$ In light of the important role of neutrophils and macrophages in host defense in pneumococcal pneumonia, ${ }^{3}$ we determined the cellular composition of BALF harvested at 6,24 or $48 \mathrm{~h}$ after infection with a lethal dose of $S$. pneumoniae $\left(10^{4} \mathrm{CFU}\right)$. Whereas, macrophage numbers were similar in BALF harvested from CD44 $\mathrm{KO}$ and WT mice at all time points (data not shown), neutrophil counts were slightly enhanced at $6 \mathrm{~h}$ and more clearly at $24 \mathrm{~h}$ after infection, although these differences did not reach statistical significance ( $P=0.16$ and $P=0.06$, respectively; Figure $3 \mathrm{a}$ ). Moreover, MPO levels were significantly increased in lung homogenates of CD44 KO as compared with WT mice at 6 but not at $24 \mathrm{~h}$ (Figure $3 \mathrm{~b}$ ), again indicating enhanced neutrophil numbers in the absence of CD44 early after lethal S. pneumoniae infection. At $48 \mathrm{~h}$ post infection, the number of neutrophils in BALF and the MPO levels in lung homogenates were similar in CD44 KO and WT mice (data not shown). To determine whether the increased neutrophil numbers might be a result of elevated 


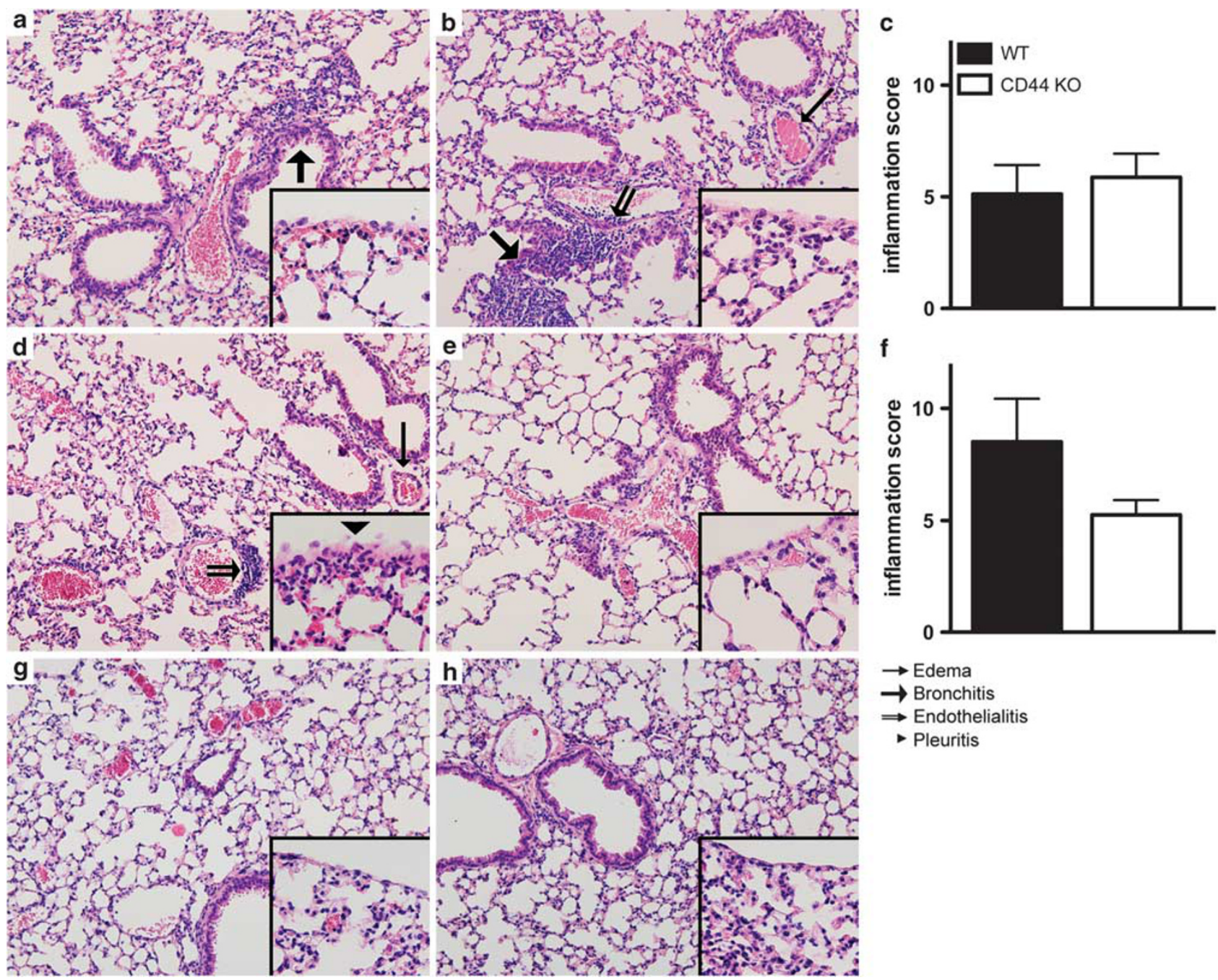

Figure 2 CD44 does not affect lung histopathology during lethal pneumonia. Representative lung pathology of WT (a, d) and CD44 KO (b, e) mice 24 (a-c), and $48(\mathbf{d}-\mathbf{f}) \mathrm{h}$ after intranasal infection with $10^{4} \mathrm{CFU}$ of $\mathrm{S}$. pneumoniae. The lung sections are representative for eight mice per group per time point. The inserts shows pleuritis. (g, h) Representative lung sections from uninfected WT $(\mathbf{g})$ and CD44 KO (h) mice. Hematoxilin and eosin staining, original magnification $\times 10$, insert $\times 40$. Quantitative data are expressed as means \pm s.e.m. of eight mice per group. WT: black bars and CD44 KO: white bars.

CXC chemokine levels, we measured LIX, KC and MIP-2 levels in lung homogenates. At $6 \mathrm{~h}$ post infection, LIX and $\mathrm{KC}$ concentrations were enhanced in lungs from $\mathrm{CD} 44 \mathrm{KO}$ mice, although for $\mathrm{KC}$ this difference did not reach statistical significance $(P<0.01$ and $P=0.08$ versus WT mice, respectively), whereas MIP-2 levels were similar in both the groups (Figure 3c-e). At 24 and $48 \mathrm{~h}$ after infection, lung CXC chemokine levels were indistinguishable in $\mathrm{WT}$ and $\mathrm{CD} 44 \mathrm{KO}$ mice (data not shown). Together, these data suggest that CD44 deficiency results in an enhanced early neutrophil influx to the primary site of infection, which at least in part might be caused by elevated lung levels of the neutrophil attracting chemokine LIX.

\section{CD44 Deficiency Facilitates Bacterial Growth and Dissemination During Sublethal Pneumonia}

The results presented above indicate that CD44 has a detrimental role in host defense during lethal pneumococcal pneumonia. Next we wished to establish the role of CD44 in antibacterial defense during sublethal pneumococcal pneumonia. In addition, considering that CD44 has been found to have an important role in the regulation of chronic lung inflammation, such as induced by intratracheal administration of bleomycin, ${ }^{12}$ we wished to study whether CD44 affects on the resolution of lung inflammation after clearance of the infection. To address these questions, we infected CD44 KO and WT mice with a low dose of S. pneumoniae $\left(10^{2} \mathrm{CFU}\right)$, and killed them either at 2,4 or 10 days after infection. At 2 days after infection, only $3 / 8$ CD 44 KO versus $6 / 7$ WT mice demonstrated bacteria in their lungs $(P=0.05)$, confirming a detrimental role of CD44 in defense against pneumococci also during sublethal respiratory tract infection. In addition, in this mild infection model, blood cultures remained sterile in CD44 KO mice, whereas in 2/7 WT mice $S$. pneumoniae could be recovered from blood; moreover, 
Table 1 Pulmonary and plasma cytokine concentrations during lethal pneumonia

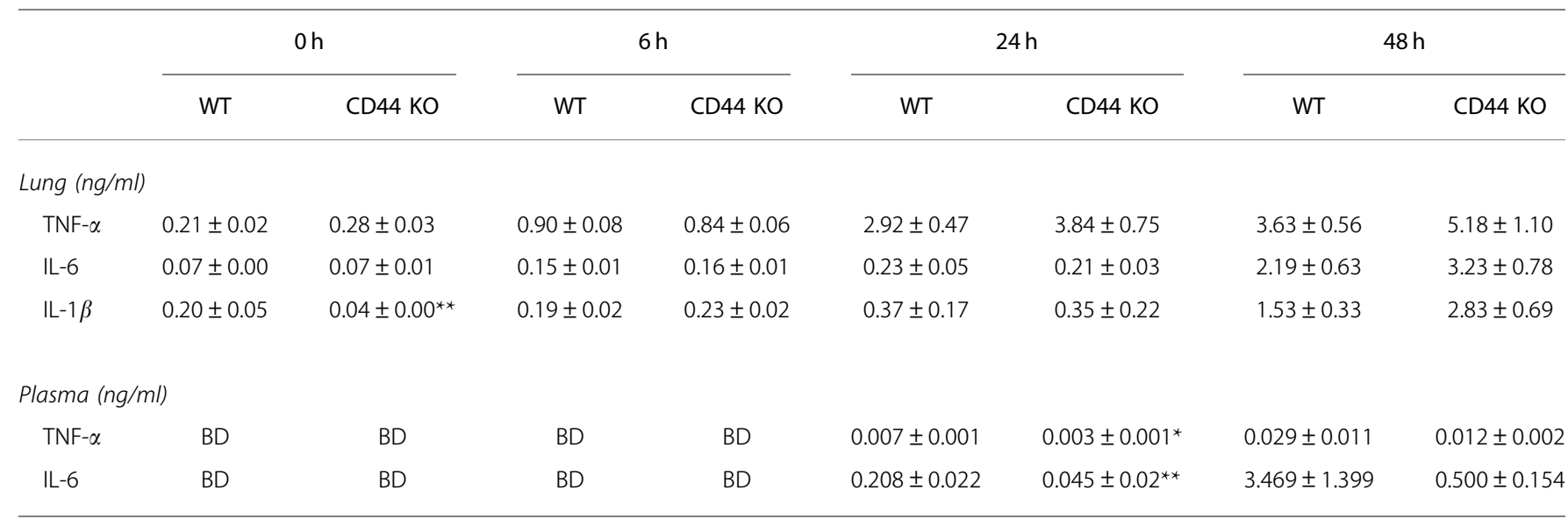

Abbreviations: BD, below detection; IL, interleukin; KO, knockout; TNF- $\alpha$, tumor necrosis factor- $\alpha$; WT, wild type.

Proinflammatory cytokine (TNF- $\alpha, \mathrm{IL}-6$ and IL-1 $\beta$ ) levels in lung and plasma at 6,24 and $48 \mathrm{~h}$ after infection with $10^{4}$ colony-forming units Streptococcus pneumoniae in WT and CD44 KO mice. Data are expressed as means \pm s.e.m.; $n=8$ mice/group per time point.

${ }^{\star} P<0.05,{ }^{*} P<0.01$ versus WT at the same time point.
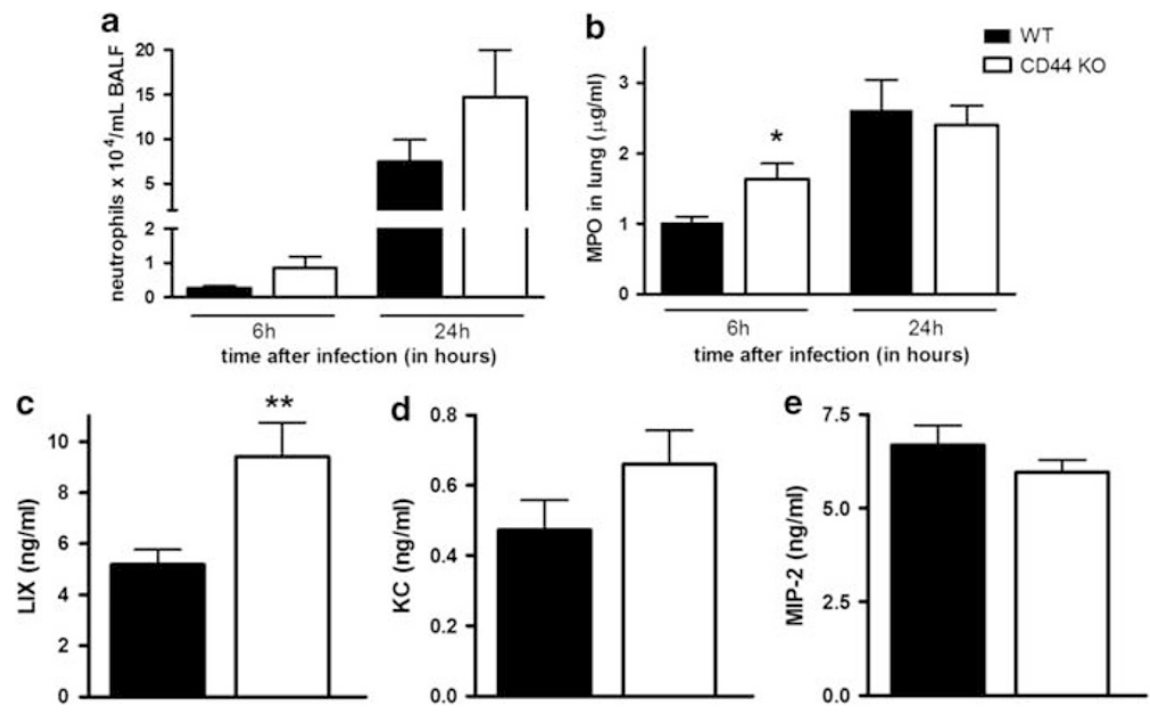

Figure 3 Enhanced early neutrophil influx and LIX levels in CD44 KO mice. Neutrophil numbers in bronchoalveolar lavage fluid (a), MPO concentrations (b) at 6 and $24 \mathrm{~h}$ after infection, and levels of LIX (c), KC (d) and MIP-2 (e) in lungs of WT (black bars) and CD44 KO (white bars) mice at $6 \mathrm{~h}$ after infection with $10^{4} \mathrm{CFU}$ of $S$. pneumoniae. Data are expressed as means \pm s.e.m.; $n=8$ mice/group, ${ }^{\star} P<0.05,{ }^{*} P<0.01$ as compared with WT mice.

spleen cultures were positive in 5/7 WT mice versus $2 / 8$ CD44 KO mice $(P=0.07)$, again (ie, such as in lethal pneumonia) hinting to a role for CD44 in facilitating bacterial dissemination. At 4 and 10 days after infection, all mice from both mouse strains had cleared the bacteria from their lungs and no bacteria could be recovered from either blood or spleen.

\section{CD44 is Important in the Resolution of Lung Inflammation During Sublethal Pneumonia}

To obtain insight into the function of CD44 in the regulation of lung inflammation after respiratory tract infection by a sublethal dose of $S$. pneumoniae, we prepared lung tissue slides and semiquantitatively scored the histopathology visualized (Figure 4). At 2 days after low-dose infection, interstitial inflammation was present in all mice, which was accompanied by endothelialitis, bronchitis, edema and/or pleuritis; lung histopathology scores did not differ between $\mathrm{CD} 44 \mathrm{KO}$ and WT mice at this time point (Figure $4 \mathrm{a}-\mathrm{c}$ ). At 4 (Figure $4 \mathrm{~d}-\mathrm{f}$ ) and 10 days (Figure $4 \mathrm{~g}-\mathrm{i}$ ) after infection, however, pulmonary inflammation scores were higher in CD44 KO mice than in WT mice ( 4 days: $P=0.13 ; 10$ days: $P<0.05)$. At 4 days, all mice showed mild edema but interstitial inflammation was more prominent in CD44 $\mathrm{KO}$ mice 

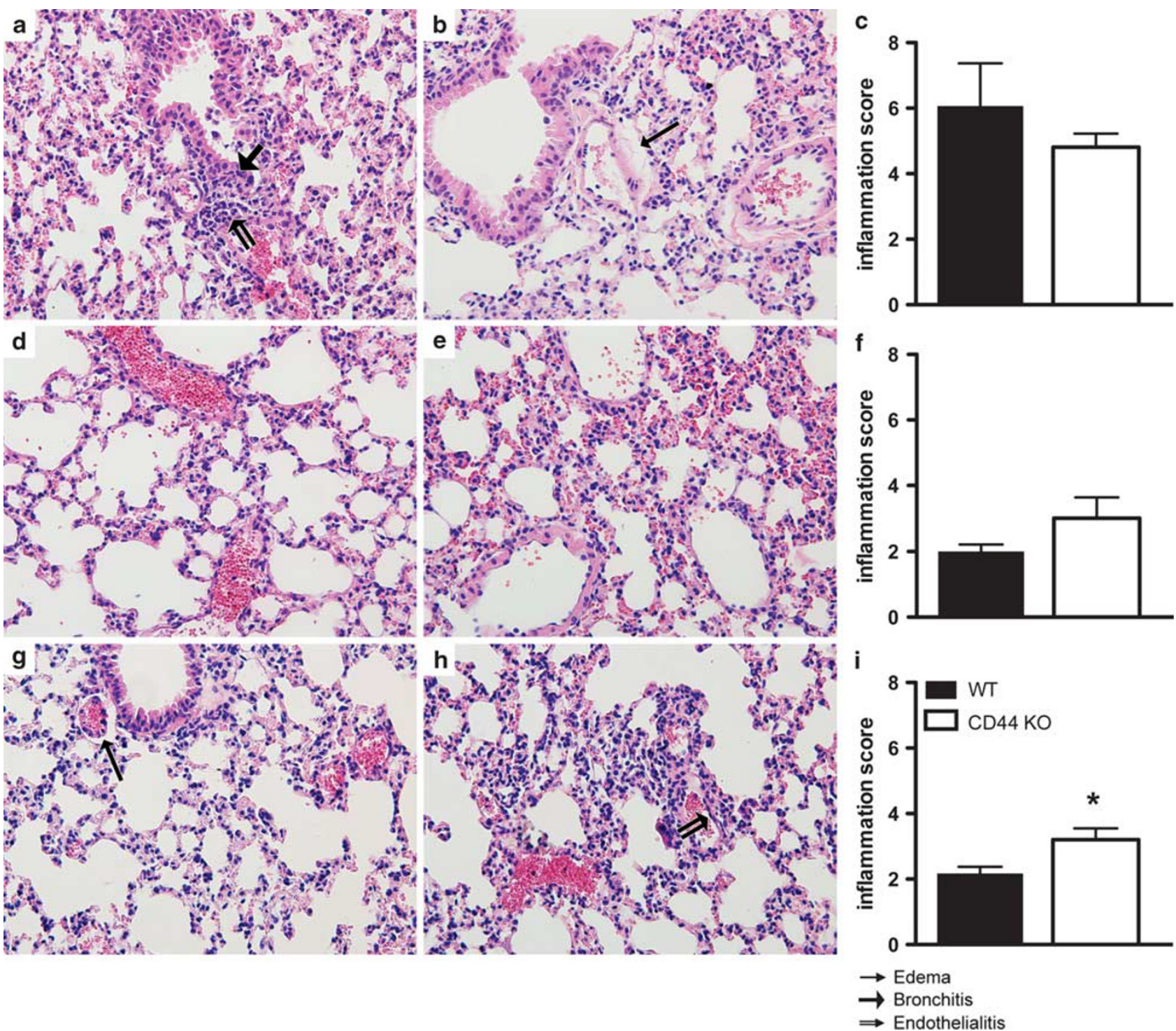

Figure 4 Prolonged lung inflammation in CD44 KO mice during sublethal pneumonia. Representative lung pathology of WT (a, d, g) and CD44 KO (b, e, h) mice at $2(\mathbf{a}-\mathbf{c}), 4(\mathbf{d}-\mathbf{f})$ and $10(\mathbf{g}-\mathbf{i})$ days after intranasal infection with $10^{2}$ CFU of S. pneumoniae. The lung sections are representative for 7-12 mice per group per time point. Hematoxilin and eosin staining, original magnification $\times 20$. Quantitative data are expressed as means \pm s.e.m. of 8-11 mice per group. WT: black bars and CD44 KO: white bars. ${ }^{*} P<0.05$ as compared with WT mice.

as compared with WT mice. At 10 days, both groups still showed significant interstitial inflammation, however, endothelialitis, bronchitis and edema were only found in lungs from CD44 KO mice. In addition, MPO levels in whole-lung homogenates (indicative for neutrophil numbers in lung tissue) were similar at 2 and 4 days, but significantly enhanced in lungs harvested from CD44 KO mice at 10 days (Table 2). In the bronchoalveolar space, CD44 deficiency was associated with much higher total leukocyte numbers at 2 days after infection, which was caused by higher neutrophil counts (Table 2, $P<0.01$ ); neutrophil numbers remained higher in BALF from CD44 KO mice at 4 days $(P<0.05)$ and 10 days of infection, although at the latter time point the difference with WT mice was not statistically significant anymore $(P=0.14)$. In addition, lymphocyte counts at 4 days were significantly higher in BALF of CD44 KO mice $(P<0.05$ versus WT mice). Macrophage numbers did not differ between mouse strains at any time point after sublethal pneumococcal infection.

\section{CD44 Deficiency is Associated with Accumulation of HA Fragments in BALF}

CD44 is known to bind and to internalize HA fragments. ${ }^{7,26}$ These fragments can induce NF- $\kappa \mathrm{B}$ activation, ${ }^{27}$ and thereby proinflammatory gene expression. ${ }^{28-31} \mathrm{CD} 44$ has been shown to have an important role in the clearance of HA from the 
Table 2 Lung myeloperoxidase concentrations and bronchoalveolar lavage fluid leukocyte numbers during sublethal Streptococcus pneumoniae infection

\begin{tabular}{|c|c|c|c|c|c|c|c|c|}
\hline & \multicolumn{2}{|c|}{0 Day } & \multicolumn{2}{|c|}{2 Days } & \multicolumn{2}{|c|}{4 Days } & \multicolumn{2}{|c|}{10 Days } \\
\hline & WT & CD44 KO & WT & CD44 KO & WT & CD44 KO & WT & CD44 KO \\
\hline \multicolumn{9}{|l|}{ Lung (ng/ml) } \\
\hline MPO & ND & ND & $1734 \pm 297$ & $1976 \pm 413$ & $737 \pm 70$ & $772 \pm 104$ & $790 \pm 72$ & $1059 \pm 97^{\star}$ \\
\hline Total & $14.88 \pm 5.38$ & $10.66 \pm 0.82$ & $9.02 \pm 0.85$ & $12.9 \pm 0.64^{* *}$ & $21.0 \pm 2.25$ & $19.1 \pm 1.51$ & $12.7 \pm 1.15$ & $11.0 \pm 0.67$ \\
\hline Neutrophils & $0.25 \pm 0.14$ & $0.19 \pm 0.13$ & $0.59 \pm 0.38$ & $3.16 \pm 0.40^{\star *}$ & $0.38 \pm 0.25$ & $0.62 \pm 0.08^{*}$ & $0.14 \pm 0.04$ & $0.64 \pm 0.25$ \\
\hline Lymphocytes & $0.28 \pm 0.06$ & $0.45 \pm 0.07$ & $0.17 \pm 0.04$ & $0.33 \pm 0.14$ & $0.06 \pm 0.04$ & $0.62 \pm 0.39^{\star}$ & $0.28 \pm 0.06$ & $0.38 \pm 0.04$ \\
\hline Macrophages & $7.65 \pm 1.47$ & $9.49 \pm 0.79$ & $8.24 \pm 0.83$ & $9.40 \pm 0.38$ & $20.5 \pm 2.20$ & $17.9 \pm 1.35$ & $11.4 \pm 1.15$ & $10.0 \pm 0.70$ \\
\hline
\end{tabular}

Abbreviations: BALF, bronchoalveolar lavage fluid; KO, knockout; MPO, myeloperoxidase; ND, not determined; WT, wild type.

MPO levels in lung and total leukocyte counts, neutrophil, lymphocyte and macrophage numbers in BALF at 2,4 and 10 days after infection with $10^{2}$ colonyforming units S. pneumoniae in WT and CD44 KO mice. Data are expressed as means \pm s.e.m.; $n=7$ to 12 mice/group per time point.

${ }^{*} P<0.05,{ }^{*} P<0.01$ versus WT at the same time point.

airways during bleomycin- or LPS-induced lung inflammation, ${ }^{8,12}$ and the accumulation of HA fragments contributes to the perpetuation of inflammation in these models. ${ }^{12,32}$ Therefore, we considered it of interest to investigate whether CD44 has a part in the clearance of HA during the resolution phase of $S$. pneumoniae-induced pneumonia. Indeed, HA levels measured in BALF obtained at 4 or 10 days after sublethal infection were much higher in CD44 KO mice than in WT mice (Figure 5). These data suggest that clearance of HA from the bronchoalveolar space is an important function of CD44 during the resolution phase of pneumococcal pneumonia.

\section{DISCUSSION}

Considering the broad functions of CD44 and its abundant expression pattern, we hypothesized that the net effect of CD44 deficiency during pneumonia is dependent on the phase and severity of the infection. This study is the first to investigate the functional role of CD44 during both the induction and resolution phase of inflammation during pneumococcal pneumonia. We here demonstrate that during lethal pneumonia, CD44 accelerates mortality and facilitates early bacterial outgrowth and dissemination. Whereas CD44 deficiency did not affect on proinflammatory cytokine responses, CD44 KO mice showed elevated pulmonary chemokine levels early after infection, which was accompanied by increased neutrophil influx into the lungs. We confirmed a detrimental role of CD44 in antibacterial defense against pneumococci also during sublethal pneumonia, and demonstrate that CD44 is important for the resolution of lung inflammation after clearance of a low dose of pneumococci.

Thus far only one study investigated the role of CD44 during bacterial pneumonia; although this investigation was

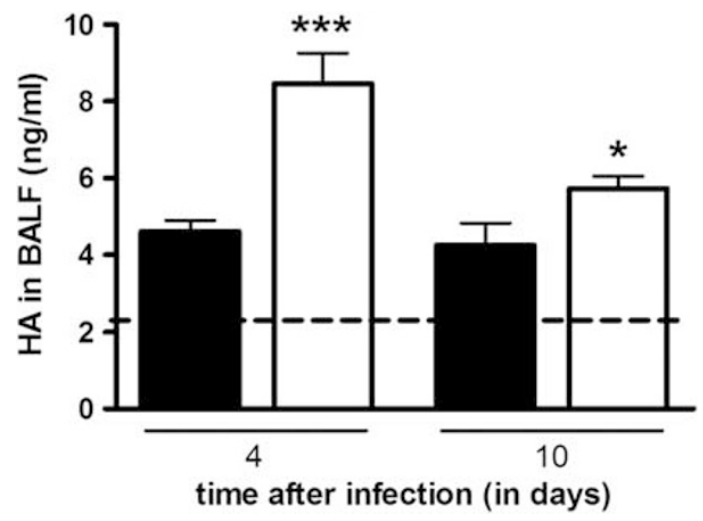

Figure 5 Elevated hyaluronic acid levels in bronchoalveolar lavage fluid from CD44 KO mice during sublethal pneumonia. Hyaluronic acid (HA) concentrations in bronchoalveolar lavage fluid (BALF) from WT (black bars) and CD44 KO (white bars) mice at 4 and 10 days after intranasal infection with $10^{2} \mathrm{CFU}$ of $S$. pneumoniae. Data are expressed as means \pm s.e.m. ${ }^{*} P<0.05,{ }^{* *} P<0.001$ versus WT at the same time point. Dashed line represents $\mathrm{HA}$ level in uninfected mice.

unable to detect any influence of CD44 on the host response in pneumococcal pneumonia, this research only examined the acute response of the first $6 \mathrm{~h} .{ }^{9}$ To obtain further insight into the role of CD44 in host defense against $S$. pneumoniae, we first used a bacterial dose that causes pneumonia, sepsis and lethality in normal immunocompetent mice. ${ }^{21,22}$ CD44 KO mice had a survival benefit relative to WT mice as demonstrated by a 2 -day longer median survival time. In concurrence, pulmonary bacterial loads and dissemination of S. pneumoniae to distant organs were reduced in CD44 KO mice at $24 \mathrm{~h}$ after infection. The detrimental role of CD44 in antibacterial defense appeared transient on infection with a lethal dose, considering that at $48 \mathrm{~h}$ bacterial numbers were 
similar in lung, blood and spleen of CD44 KO and WT mice. Conceivably, the unfavorable effect of CD44 during pneumococcal pneumonia depends on the stage of the infection, becoming less important as the growing bacterial load overwhelms innate immune defense mechanisms, such as occurs during late stage lethal infection. In support of this notion, CD44 KO mice displayed evidence for an improved antibacterial defense at 2 days after infection with a sublethal dose of pneumococci, as reflected by the fact that 5/8 CD44 $\mathrm{KO}$ mice had cleared S. pneumoniae from their lungs versus only $1 / 7$ WT mice, which also was associated with a diminished dissemination to blood and spleen in CD44 $\mathrm{KO}$ mice. The reduced bacterial loads in CD44 KO mice seem counterintuitive considering the previously described role for CD44 in phagocytosis of bacteria such as M. tuberculosis ${ }^{13}$ and S. aureus. ${ }^{14}$ However, in preliminary experiments, we could not identify such a role for CD44 in phagocytosis of S. pneumoniae (data not shown). Moreover, increased dissemination of pneumococci in the presence of CD44 could in theory also be a result of the bacteria using CD44 to overcome the epithelial-endothelial barrier, a mechanism that has previously been described for pneumocci and the plateletactivating factor receptor, ${ }^{33,34}$ but also for urothelial CD44 and E. coli through binding of HA. ${ }^{11}$ However, we could not demonstrate an interaction between CD44 and S. pneumoniae either directly or indirectly through HA (data not shown).

CD44 did not affect on pulmonary pathology during lethal pneumonia. In addition, we found no differences in the pulmonary levels of proinflammatory cytokines between WT and $\mathrm{CD} 44 \mathrm{KO}$ mice, suggesting that CD44 is not involved in the regulation of the acute inflammatory response in the lung in the first $48 \mathrm{~h}$ after a lethal challenge with S. pneumoniae via the airways. For several stimuli, CD44 has been implicated to affect proinflammatory cytokine release. For instance, CD44 KO mice demonstrated increased IL- $1 \beta$ and TNF- $\alpha$ levels in their BALF on a pulmonary LPS challenge, ${ }^{8}$ and CD44 KO bone marrow-derived macrophages produced more TNF- $\alpha$ and IL- 6 on stimulation with not only LPS but also with ligands for Toll-like receptor (TLR)3, TLR5, TLR7 or TLR9. ${ }^{35}$ In these studies, however, single ligands for specific TLRs were used, whereas intact pneumococci express multiple ligands that can stimulate several pathways resulting in cytokine release. Indeed, ex vivo stimulation of CD44 KO alveolar macrophages with $S$. pneumoniae did not result in different TNF- $\alpha$ release as compared with WT cells (data not shown). Of note, in plasma we found decreased levels of TNF- $\alpha$ and IL-6 in CD44 KO mice at $24 \mathrm{~h}$ after infection, which most likely was related to a reduced systemic proinflammatory stimulus for enhanced cytokine release at this time point because of the fact that only $1 / 8 \mathrm{CD} 44 \mathrm{KO}$ mice versus $8 / 8$ WT mice these animals demonstrated positive blood cultures.

Several studies have shown a positive role of CD44 in inflammatory cell recruitment, ${ }^{10,25,36-41}$ whereas other investigations reported enhanced inflammatory cell migration in the absence of CD44. ${ }^{8,9,24,35}$ This discrepancy is probably because of the differences in the cell type studied, the stimulus and organ involved, and the severity of the insult. We here found no differences in macrophage numbers in BALF of CD44 KO and WT mice during either lethal or sublethal pneumococcal pneumonia, indicating that CD44 does not affect on macrophage recruitment into the bronchoalveolar space induced by S. pneumoniae. Of note, in models of sterile lung inflammation elicited by intrapulmonary delivery of either LPS or bleomycin CD44 KO mice demonstrated increased macrophage numbers in their BALF, ${ }^{8,12}$ whereas during pulmonary tuberculosis CD44 KO mice had lower macrophage counts in their lungs, ${ }^{13}$ exemplifying the complex role of $\mathrm{CD} 44$ in macrophage trafficking toward the lungs. We did observe increased neutrophil numbers in the lungs of CD44 KO mice during S. pneumoniae pneumonia. During lethal infection, MPO levels were higher in wholelung homogenates after $6 \mathrm{~h}$, indicative for a higher neutrophil content of lung tissue; in BALF neutrophil counts tended to be higher in CD44 KO mice, especially at $24 \mathrm{~h}(P=0.06$ versus WT mice); these data suggest that CD44 deficiency was associated with an increased influx of neutrophils to the pulmonary compartment early after infection, first in lung tissue (as reflected by MPO levels in whole-lung homogenates) and subsequently in the bronchoalveolar space (as reflected by neutrophil counts in BALF). During sublethal pneumonia, the number of neutrophils in BALF of CD44 KO mice was significantly higher at $2-4$ days post infection. An earlier study reported unaltered neutrophil counts in BALF obtained at $6 \mathrm{~h}$ after intratracheal infection with an unspecified clinical isolate of $S$. pneumoniae, ${ }^{9}$ which is not necessarily in contrast with our current results. In lung infection caused by E. coli ${ }^{9}$ or M. tuberculosis, ${ }^{13}$ CD44 deficiency also resulted in increased neutrophil numbers in the pulmonary compartment. The mechanism by which CD44 affects on pulmonary neutrophil counts during infectious lung inflammation remains to be established. CD44 deficiency may positively influence neutrophil recruitment: pathogen-derived molecules, such as $\mathrm{N}$-formyl-met-leu-phe (fMLP), are potent chemoattractants for neutrophils during pneumococcal pneumonia, ${ }^{42}$ and migration of CD44 KO neutrophils through Matrigel toward a fMLP gradient was enhanced when compared with migration of WT neutrophils. ${ }^{9}$ The recruitment of neutrophils in CD44 KO mice could have been further facilitated by increased pulmonary levels of the neutrophil attracting CXC chemokines LIX and to a lesser extent KC early after infection. Alternatively, in bleomycin-induced lung inflammation, macrophage CD44 has an important role in the clearance of apoptotic neutrophils, at least in part explaining the neutrophil accumulation in this model. ${ }^{12}$

CD44 has been found to have an important role in the regulation of chronic lung inflammation, such as induced by intratracheal administration of bleomycin. ${ }^{12}$ The majority of patients suffering from pneumococcal pneumonia recovers; ${ }^{43}$ 
in these patients the resolution of lung inflammation is an important part of an adequate host response. Therefore, we additionally studied the role of CD44 during sublethal pneumonia using an inoculum that is cleared by WT mice in several days. Our data provide evidence that CD44 contributes to the resolution of lung inflammation after clearance of the infection. Indeed, whereas pulmonary inflammation did not differ between CD44 KO and WT mice after 2 and 4 days of infection, after 10 days CD44 KO mice demonstrated enhanced lung inflammation scores. CD44 is known not only to bind but also to internalize HA fragments. $^{7,26}$ These fragments can induce NF- $\kappa \mathrm{B}$ activation, ${ }^{27}$ and thereby proinflammatory gene expression through TLR2 and TLR4 signaling. ${ }^{28-31}$ Indeed, CD44 KO mice showed impaired clearance of $\mathrm{HA}$ in bleomycin-induced lung inflammation accompanied by sustained accumulation of inflammatory cells. ${ }^{12}$ In line, we found significantly increased HA concentrations in BALF from CD44 KO mice at 4 and 10 days after sublethal pneumonia, suggesting that, such as in bleomycin-induced lung inflammation, CD44 facilitates the resolution of inflammation during pneumococcal pneumonia, at least in part through clearance of HA from the lungs.

In conclusion, we show here that CD44 influences several components of the innate immune response to $S$. pneumoniae in the airways using two different infectious doses, one lethal and one non-lethal. In the first set of experiments, using a lethal dose of $S$. pneumoniae, we documented an improved antibacterial defense, as reflected by a reduced bacterial growth in the lung and a diminished dissemination to blood at $24 \mathrm{~h}$ after infection, subsequently associated with a prolonged survival. Although our studies do not provide a clear mechanism for the beneficial phenotype of CD44 KO mice, the enhanced neutrophil influx very early after lethal infection may have played a role. ${ }^{3}$ During these lethal infection studies, CD44 did not affect on lung pathology, arguing against a role of importance for this receptor in the induction of the histopathological pulmonary changes that accompany acute severe pneumococcal pneumonia. The second set of experiments focused on the resolution phase of non-lethal pneumococcal pneumonia. In this study, the unfavorable role of CD44 in bacterial growth and dissemination was confirmed. In addition, CD44 was demonstrated to contribute to an adequate resolution of the inflammatory response in the lung after clearance of pneumococci (4 or 10 days after infection). Together, these data suggest that CD44 facilitates bacterial growth and dissemination during lethal and non-lethal pneumococcal pneumonia, whereas it promotes the resolution of lung inflammation during mild pneumococcal pneumonia after clearance of the causative pathogen.

\section{ACKNOWLEDGEMENT}

We thank Joost Daalhuisen, Marieke ten Brink and Regina de Beer for expert technical assistance.

\section{DISCLOSURE/CONFLICT OF INTEREST}

The authors declare no conflict of interest.

1. Armstrong GL, Conn LA, Pinner RW. Trends in infectious disease mortality in the United States during the 20th century. JAMA 1999;281:61-66.

2. Mandell LA, Wunderink RG, Anzueto A, et al. Infectious Diseases Society of America/American Thoracic Society consensus guidelines on the management of community-acquired pneumonia in adults. Clin Infect Dis 2007;44(Suppl 2):S27-S72.

3. van der Poll T, Opal SM. Pathogenesis, treatment, and prevention of pneumococcal pneumonia. Lancet 2009;374:1543-1556.

4. Campbell Jr GD, Silberman R. Drug-resistant Streptococcus pneumoniae. Clin Infect Dis 1998;26:1188-1195.

5. Isacke CM, Yarwood $\mathrm{H}$. The hyaluronan receptor, CD44. Int J Biochem Cell Biol 2002;34:718-721.

6. Pure E, Cuff CA. A crucial role for CD44 in inflammation. Trends Mol Med 2001;7:213-221.

7. Culty M, Nguyen HA, Underhill CB. The hyaluronan receptor (CD44) participates in the uptake and degradation of hyaluronan. J Cell Biol 1992;116:1055-1062.

8. Liang J, Jiang D, Griffith J, et al. CD44 is a negative regulator of acute pulmonary inflammation and lipopolysaccharide-TLR signaling in mouse macrophages. J Immunol 2007;178:2469-2475.

9. Wang $Q$, Teder $P$, Judd NP, et al. CD44 deficiency leads to enhanced neutrophil migration and lung injury in Escherichia coli pneumonia in mice. Am J Pathol 2002;161:2219-2228.

10. Rouschop KM, Roelofs JJ, Claessen N, et al. Protection against renal ischemia reperfusion injury by CD44 disruption. J Am Soc Nephrol 2005;16:2034-2043.

11. Rouschop KM, Sylva M, Teske GJ, et al. Urothelial CD44 facilitates Escherichia coli infection of the murine urinary tract. J Immunol 2006;177:7225-7232.

12. Teder $\mathrm{P}$, Vandivier RW, Jiang $\mathrm{D}$, et al. Resolution of lung inflammation by CD44. Science 2002;296:155-158.

13. Leemans JC, Florquin S, Heikens $M$, et al. CD44 is a macrophage binding site for Mycobacterium tuberculosis that mediates macrophage recruitment and protective immunity against tuberculosis. J Clin Invest 2003;111:681-689.

14. Moffat Jr FL, Han T, Li ZM, et al. Involvement of CD44 and the cytoskeletal linker protein ankyrin in human neutrophil bacterial phagocytosis. J Cell Physiol 1996;168:638-647.

15. Serhan CN, Brain SD, Buckley CD, et al. Resolution of inflammation: state of the art, definitions and terms. FASEB J 2007;21:325-332.

16. Mizgerd JP. Acute lower respiratory tract infection. N Engl J Med 2008;358:716-727.

17. Schmits R, Filmus J, Gerwin N, et al. CD44 regulates hematopoietic progenitor distribution, granuloma formation, and tumorigenicity. Blood 1997;90:2217-2233.

18. Knapp S, Wieland CW, van 't Veer C, et al. Toll-like receptor 2 plays a role in the early inflammatory response to murine pneumococcal pneumonia but does not contribute to antibacterial defense. J Immunol 2004;172:3132-3138.

19. Dessing MC, Knapp S, Florquin S, et al. CD14 facilitates invasive respiratory tract infection by Streptococcus pneumoniae. Am J Respir Crit Care Med 2007;175:604-611.

20. Dessing MC, Florquin S, Paton JC, et al. Toll-like receptor 2 contributes to antibacterial defence against pneumolysin-deficient pneumococci. Cell Microbiol 2008;10:237-246.

21. Stegenga $M E$, Florquin $S$, de $\operatorname{Vos} A F$, et al. The thiazolidinedione ciglitazone reduces bacterial outgrowth and early inflammation during Streptococcus pneumoniae pneumonia in mice. Crit Care Med 2009;37:614-618.

22. van Zoelen MA, Schouten $M$, de Vos AF, et al. The receptor for advanced glycation end products impairs host defense in pneumococcal pneumonia. J Immunol 2009;182:4349-4356.

23. Strieter RM, Belperio JA, Keane MP. Host innate defenses in the lung: the role of cytokines. Curr Opin Infect Dis 2003;16:193-198.

24. Si-Tahar M, Sitaraman S, Shibahara $T$, et al. Negative regulation of epithelium-neutrophil interactions via activation of CD44. Am J Physiol Cell Physiol 2001;280:C423-C432. 
25. Alstergren $\mathrm{P}$, Zhu $\mathrm{B}$, Glogauer $\mathrm{M}$, et al. Polarization and directed migration of murine neutrophils is dependent on cell surface expression of CD44. Cell Immunol 2004;231:146-157.

26. Harada H, Takahashi M. CD44-dependent intracellular and extracellular catabolism of hyaluronic acid by hyaluronidase- 1 and -2 . J Biol Chem 2007;282:5597-5607.

27. Noble PW, McKee CM, Cowman $M$, et al. Hyaluronan fragments activate an NF-kappa B/I-kappa B alpha autoregulatory loop in murine macrophages. J Exp Med 1996;183:2373-2378.

28. Voelcker V, Gebhardt C, Averbeck M, et al. Hyaluronan fragments induce cytokine and metalloprotease upregulation in human melanoma cells in part by signalling via TLR4. Exp Dermatol 2008;17:100-107.

29. Taylor KR, Trowbridge JM, Rudisill JA, et al. Hyaluronan fragments stimulate endothelial recognition of injury through TLR4. J Biol Chem 2004;279:17079-17084.

30. Scheibner KA, Lutz MA, Boodoo $S$, et al. Hyaluronan fragments act as an endogenous danger signal by engaging TLR2. J Immunol 2006; 177:1272-1281.

31. Jiang D, Liang J, Fan J, et al. Regulation of lung injury and repair by Toll-like receptors and hyaluronan. Nat Med 2005;11:1173-1179.

32. Savani RC, Hou G, Liu $P$, et al. A role for hyaluronan in macrophage accumulation and collagen deposition after bleomycin-induced lung injury. Am J Respir Cell Mol Biol 2000;23:475-484.

33. Cundell DR, Gerard NP, Gerard C, et al. Streptococcus pneumoniae anchor to activated human cells by the receptor for platelet-activating factor. Nature 1995;377:435-438.
34. Rijneveld AW, Weijer S, Florquin S, et al. Improved host defense against pneumococcal pneumonia in platelet-activating factor receptordeficient mice. J Infect Dis 2004;189:711-716.

35. Kawana $H$, Karaki $H$, Higashi $M$, et al. CD44 suppresses TLR-mediated inflammation. J Immunol 2008;180:4235-4245.

36. Hollingsworth JW, Li Z, Brass DM, et al. CD44 regulates macrophage recruitment to the lung in lipopolysaccharide-induced airway disease. Am J Respir Cell Mol Biol 2007;37:248-253.

37. McDonald B, McAvoy EF, Lam F, et al. Interaction of CD44 and hyaluronan is the dominant mechanism for neutrophil sequestration in inflamed liver sinusoids. J Exp Med 2008;205:915-927.

38. Kimura K, Nagaki $M$, Kakimi $K$, et al. Critical role of CD44 in hepatotoxin-mediated liver injury. J Hepatol 2008;48:952-961.

39. Gonda A, Gal I, Szanto S, et al. CD44, but not I-selectin, is critically involved in leucocyte migration into the skin in a murine model of allergic dermatitis. Exp Dermatol 2005;14:700-708.

40. Khan Al, Kerfoot SM, Heit B, et al. Role of CD44 and hyaluronan in neutrophil recruitment. J Immunol 2004;173:7594-7601.

41. DeGrendele HC, Estess P, Siegelman MH. Requirement for CD44 in activated $\mathrm{T}$ cell extravasation into an inflammatory site. Science 1997;278:672-675.

42. Fillion I, Ouellet N, Simard $M$, et al. Role of chemokines and formyl peptides in pneumococcal pneumonia-induced monocyte/macrophage recruitment. J Immunol 2001;166:7353-7361.

43. Restrepo MI, Anzueto A. Severe community-acquired pneumonia. Infect Dis Clin North Am 2009;23:503-520. 